\title{
Quality of Life in Younger versus Older Breast Cancer Survivors
}

\author{
Kim Ziner, PhD $^{1}$ \\ Victoria Champion, RN, $\mathbf{P h D}^{1}$ \\ George Sledge, $\mathrm{MD}^{2}$ \\ Patrick Monahan, $\mathbf{P h D}^{2}$ \\ Qian Qian Zho, MS $^{2}$ \\ ${ }^{1}$ Indiana University School of Nursing
${ }^{2}$ Indiana University School of Medicine \\ Indiana University - Purdue University Indianapolis
}

\begin{abstract}
Background: Breast cancer is one of the most frequently occurring cancers in the developing world, but with earlier detection and better treatment, the majority of breast cancer survivors will live many years after diagnosis. Breast cancer survivors may experience many symptoms that impact their quality of life, and these symptoms may vary by age. The purpose of this study is to compare breast cancer survivors who were diagnosed at 45 and under $(n=469)$ with survivors diagnosed at 55 to $70(n=584)$ years of age.

Materials and methods: Participants were identified through a large cooperative group (Eastern Cancer Cooperative Group). Eligibility criteria included use of chemotherapy at initial diagnosis, being 3 to 8 years from diagnosis, and not having a recurrence of breast cancer. The mean current age of younger survivors was 45.2 and for older survivors was 66.7. Women who agreed to participate were sent a survey and informed consent which was completed and returned via mail. Overall, $80 \%$ of eligible women contacted by researchers agreed to participate. Measures included physical, psychological, social, spiritual, and overall quality of life constructs. All measurements had good reported validity and reliability. A total of 469 younger and 584 older breast cancer survivors are included. Linear regression was used to compare the two groups on continuous outcomes while adjusting for the following potentially confounding covariates: marital status (married versus not), years of education, and total household income, and years since diagnosis.

Results: Younger survivors scored significantly worse than older survivors on gynecological problems, sexual enjoyment, attention function, and overall reported symptoms.

Psychologically, younger survivors demonstrated greater symptom distress, greater depression, and greater state and trait anxiety than older survivors. Younger survivors had lower marital satisfaction scores. Younger survivors reported greater fear of recurrence and less favorable body image. Younger survivors reported lower perceived social support from their partners and greater social constraint. Older survivors held higher spiritual beliefs and behaviors as compared to younger survivors. Perceived self efficacy for dealing with problems related to cancer survivorship was lower in younger survivors as compared to older survivors. For overall quality of life measures, younger survivors reported lower index of well being scores than older survivors and reported that breast cancer had a greater impact on their life. Health care service use was greater for younger as opposed to older survivors both during and after treatment. Conclusions: Younger survivors reported significantly more problems on several, physical, psychological, social and generic quality of life issues as compared to older survivors. Results indicate a need to proactively assess quality of life issues in younger women at time of diagnosis.
\end{abstract}

